\title{
Fiberoptic endoscopic evaluation of swallowing in intensive care unit patients
}

\author{
Gert Hafner $\cdot$ Andreas Neuhuber $\cdot$ Sylvia Hirtenfelder $\cdot$ \\ Brigitte Schmedler · Hans Edmund Eckel
}

Received: 10 May 2007 / Accepted: 11 October 2007 / Published online: 30 October 2007

(C) Springer-Verlag 2007

\begin{abstract}
Aspiration in critically ill patients frequently causes severe co-morbidity. We evaluated a diagnostic protocol using routine FEES in critically ill patients at risk to develop aspiration following extubation. We instructed intensive care unit physicians on specific risk factors for and clinical signs of aspiration following extubation in critically ill patients and offered bedside FEES for such patients. Over a 45 -month period, we were called to perform 913 endoscopic examinations in 553 patients. Silent aspiration or aspiration with acute symptoms (cough or gag reflex as the bolus passed into the trachea) was detected in $69.3 \%$ of all patients. Prolonged non-oral feeding via a naso-gastric tube was initiated in $49.7 \%$ of all patients. In $13.2 \%$ of patients, a percutaneous endoscopic gastrostomy was initiated as a result of FEES findings, and in $6.3 \%$ an additional tracheotomy to prevent aspiration had to be initiated. In 59 out of 258 patients ( $22.9 \%$ ), tracheotomies were closed, and $30.7 \%$ of all 553 patients could be managed with the immediate onset of an oral diet and compensatory treatment procedures. Additional radiological examinations were not required. FEES in critically ill patients allows for a rapid evaluation of deglutition and for the immediate initiation of symptom-related rehabilitation or for an early resumption of oral feeding.
\end{abstract}

Presented at the sixth Congress of the European Laryngological Society, Nottingham, Great Britain, 30 August 2006 to 2 September 2006.

G. Hafner $\cdot$ A. Neuhuber $\cdot$ S. Hirtenfelder $\cdot$ B. Schmedler

H. E. Eckel $(\square)$

Department of Oto-Rhino-Laryngology,

Klagenfurt General Hospital, A.ö. Landeskrankenhaus Klagenfurt,

HNO, St. Veiter Str. 47, 9027 Klagenfurt, Austria

e-mail: hans.eckel@kabeg.at
Keywords Aspiration · Deglutition · Swallowing ·

Dysphagia · Tracheotomy · Rehabilitation · Endoscopy · Laryngology

\section{Introduction}

Pulmonary aspiration is a serious cause of morbidity and mortality in patients with a depressed sensorium, patients with neuromuscular discoordination, or patients having structural disorders of the upper aerodigestive tract. It is a leading cause of nosocomial infection in the intensive care unit (ICU). The most common manifestations of pulmonary aspiration are pneumonia, pleuropulmonary infection, and acute airway obstruction [1].

Hospital-acquired pneumonia is the second most common nosocomial infection in the critically ill patient and is associated with the greatest mortality and increased morbidity and cost of care. Incidence of HAP varies in different populations of critically ill patients and generally ranges from 9 to $20 \%$. The etiologic agents differ according to the population studied, duration of hospital stay, time after intubation, and prior antimicrobial therapy [2]. Risk factors include nonmodifiable factors like age, chronic obstructive pulmonary disease, severe head trauma, and multiple trauma, and modifiable factors like large-volume gastric aspiration, duration of mechanical ventilation, elevated gastric $\mathrm{pH}$, histamine type two blocker therapy, ventilator circuit change frequency, self-extubation, and silent aspiration following scheduled extubation [2]. When patients are admitted to the ICU, they are at increased risk of more frequent aspiration events because of a variety of factors, including depressed level of consciousness (often caused by excess analgesia and sedation), forced supine position, and the presence of nasogastric or endotracheal tubes [3]. 
There is a growing body of evidence suggesting that intubation for longer than $48 \mathrm{~h}$ may cause at least transient injury to the larynx with a subsequent reduction in the protective mechanism and increased incidence of oropharyngeal secretions once the patient is extubated. The presence of an orotracheal tube has been shown to alter the mechanoreceptors and chemoreceptors of the pharyngeal and laryngeal mucosa, causing dysfunction of the swallowing reflex. The prevalence of swallowing dysfunction post-extubation has been reported to occur in between 20 and $83 \%$ of those patients intubated longer than $48 \mathrm{~h}$. This wide range of estimate has partly been attributed to the variation in the diagnostic tools [4]. The impact that diagnosis using invasive diagnostic techniques may have on the epidemiological characteristics of HAP in critically ill patients is unknown, but may potentially improve aspiration-related specific therapy and ultimately clinical outcome.

Based on these considerations, we prospectively evaluated a diagnostic protocol using routine fiberoptic endoscopic evaluation of swallowing (FEES) in critically ill patients at risk to develop aspiration following temporary transnasal intubation or tracheotomy and tried to define the impact of post-extubation FEES on the initiation of aspiration-related treatment.

\section{Material and methods}

We conducted a prospective, interventional, clinical study at Klagenfurt General Hospital. The hospital is a 1,400-bed tertiary referral centre that serves a population of approximately 1,000,000 and runs seven ICUs (paediatrics and neonatology, 1; neurology, 1; neurosurgery, 1; cardiology, 1; general internal medicine, 1; and anaesthesiology, 2) with a total of 84 beds.

We offered a standardized endoscopy protocol for critically ill patients considered to be at risk for silent aspiration following transnasal intubation or tracheotomy for ventilation. Patient selection for endoscopic assessment of swallowing was done by ICU physicians using a protocol that we designed to help in the early recognition of risk factors for, or overt signs of, aspiration. This protocol included a taxonomic summary of symptoms and clinical signs potentially related to aspiration. Rating was applied after the stop of mechanical ventilation. Symptoms were defined as:

- Weight loss,

- unexplained fever $>38.0^{\circ}$ centigrade,

- coughing,

- bronchitis/pneumonitis,

- impaired voice,
- witnessed regurgitation/aspiration event at the bedside accompanied by coughing, choking, and/or expectoration of material,

- prolonged oral feeding,

- aversion for oral intake of liquids and solids,

- disturbance of bolus transport,

- frequent postural changes during oral intake, and

- regurgitation.

If such conditions were observed in previously intubated patients following extubation, or in patients with tracheotomies, physicians at ICUs were encouraged to request endoscopic assessment of swallowing.

The FEES procedure was consequently provided by staff members of the department of Oto-Rhino-Laryngology, Head and Neck Surgery. The team consisted of a laryngologist and a nurse who had both previously gone through a specialized training in performing FEES procedures in patients with deglutition disorders.

Over the 45-month period from 1 January 2002 to 30 September 2005, we were called to perform 913 endoscopic examinations in 553 patients treated in one of the ICUs. Four hundred and 46 patients underwent one single endoscopy, and 107 had repeated examinations. Two hundred and 95 patients had shortly before been extubated after transnasal tracheal intubation, and 258 patients had indwelling tracheotomies. For further data interpretation, patients were sub-grouped according to their route of ventilation (transoral/transnasal intubation vs. tracheotomy). Findings and recommendations from the initial FEES procedure in all 553 patients were accumulated for this study. The results of 360 control procedures were not further analyzed.

Details of the FEES procedure have previously been described in detail. Therefore, only a short summary of the procedure shall be given here: The FEES procedure is a portable examination, easily taken to bedside in ICUs. Since our patients were usually bedridden, we performed the examination in a in bed with the head of bed elevated to approximately $70^{\circ}$ with the bend of the bed is at the patient's lower back (904/913 patients, i.e., 99\%). In four patients $(0.4 \%)$, the examination was carried out with the patient in reverse Trendelenberg at $30^{\circ}-45^{\circ}$, and five patients $(0.6 \%)$ were able to sit on a chair during the procedure. A fiberoptic laryngoscope was passed transnasally to the oropharynx, where the larynx and surrounding structures could then be visualized. Patients were led through various tasks to evaluate the sensory and motor status of the pharyngeal and laryngeal mechanism. Stained liquid and semi-liquid boluses were then given to determine the integrity of pharyngeal deglutition. The interior larynx and airway were examined for evidence of food penetration within the laryngeal vestibule and aspiration of food below the 
true vocal folds before and after each swallow. In each case, the nasogastric tube was removed prior to the procedure. We assessed structural changes of the larynx and pharynx, timing and direction of movement of the bolus through the pharynx, the ability to protect the airway and to uphold airway protection for a some seconds, the capability to clear the bolus during deglutition, presence of pooling and residue of material in the hypopharynx, and timing of bolus flow and laryngeal closure.

Aspiration was defined as the entry of material into the airway below the levels of the true vocal cords. Silent aspiration was defined as aspiration occurring in the absence of acute symptoms (i.e., lack of cough or gag reflex as the food or liquid bolus passed into the trachea).

Findings were documented in a standardized form to allow for monitoring therapeutic interventions over time, and for later data analysis and evaluation.

\section{Results}

Over a 45-month period, we were called to perform 913 endoscopic examinations in 553 patients. Of these, 295 patients had previously been extubated, and 258 patients had indwelling tracheotomies. Silent aspiration or aspiration with acute symptoms (cough or gag reflex as the bolus passed into the trachea) was detected in 383 $(69.3 \%)$ of all patients during initial FEES. Prolonged non-oral feeding via a naso-gastric tube was initiated in $49.7 \%$ of all patients. In $13.2 \%$ of patients, a percutaneous endoscopic gastrostomy was initiated as a result of FEES findings, and in $6.3 \%$ an additional tracheotomy to prevent aspiration had to be set off. In 59 out of 258 patients (22.9\%), tracheotomies were closed, and $30.7 \%$ of 553 patients could be managed with the immediate onset of an oral diet \pm compensatory treatment procedures without further diagnostic evaluation. Logopedic instruction of the patient and/or rehabilitation using compensatory strategies to reduce the risk of aspiration were an integral part of our rehabilitation program and included one or more of the following:

- Modifications in volume and tempo of food presentation

- oral feeding with consistency modifications

- head rotation

- holding the chin down during deglutition to narrow the airway entrance

- supraglottic swallow: This technique uses simultaneous swallowing and breath-holding, closing the vocal cords and protecting the airway.

One hundred and seven patients were scheduled for repeated FEES, usually to assess the progress of the rehabilitation program.
Signs and symptoms encountered during initial FEES are condensed in Table 1. Table 2 stratifies the route of feeding in the two subgroups of our study population. Table 3 classifies the type of aspiration detected during FEES. Table 4 summarizes the recommendations derived from our endoscopic findings for the further management of patients with aspiration.

Table 1 Symptoms of aspiration in 553 patients

\begin{tabular}{lll}
\hline & $\begin{array}{l}\text { Patients after } \\
\text { transnasal } \\
\text { tracheal intubation } \\
(n=295)\end{array}$ & $\begin{array}{l}\text { Patients with } \\
\text { indwelling } \\
\text { tracheotomies } \\
(n=258)\end{array}$ \\
\hline $\begin{array}{l}\text { Unexplained fever } \\
\text { Coughing }\end{array}$ & $17(5.8 \%)$ & $39(15.1 \%)$ \\
Bronchitis/pneumonitis & $68(23.1 \%)$ & $31(12.0 \%)$ \\
Impaired voice & $57(19.32 \%)$ & $39(15.1 \%)$ \\
Witnessed regurgitation/ & $74(25.1 \%)$ & $0(0 \%)$ \\
aspiration event & $16(5.4 \%)$ & $149(57.8 \%)$ \\
Others & $69(23.3 \%)$ & $23(8.9 \%)$ \\
No data & $19(7.4 \%)$ \\
\hline
\end{tabular}

Since more than one symptom or sign could apply per patient, results sum up to more than $100 \%$

Table 2 Route of feeding for 553 patients on initial FEES

\begin{tabular}{lll}
\hline & $\begin{array}{l}\text { Patients after } \\
\text { transnasal tracheal } \\
\text { intubation } \\
(n=295)\end{array}$ & $\begin{array}{l}\text { Patients with } \\
\text { indwelling } \\
\text { tracheotomies } \\
(n=258)\end{array}$ \\
\hline $\begin{array}{l}\text { Nasogastric } \\
\text { tube }\end{array}$ & $224(75.9 \%)$ & $186(72.1 \%)$ \\
PEG & $15(5.1 \%)$ & $59(22.9 \%)$ \\
Parenteral & $12(4.1 \%)$ & $5(1.9 \%)$ \\
Oral diet & $44(14.9 \%)$ & $8(3.1 \%)$ \\
\hline
\end{tabular}

$P E G$ percutaneous endoscopic gastrostomy

Table 3 Classification of aspiration for 553 patients on initial FEES

\begin{tabular}{lll}
\hline & $\begin{array}{l}\text { Patients after } \\
\text { transoral or transnasal } \\
\text { tracheal intubation } \\
(n=295)\end{array}$ & $\begin{array}{l}\text { Patients with } \\
\text { indwelling } \\
\text { tracheotomies } \\
(n=258)\end{array}$ \\
\hline Silent aspiration & $51(17.3 \%)$ & $95(36.8 \%)$ \\
Aspiration & $115(39.0 \%)$ & $126(48.8 \%)$ \\
No aspiration & $129(43.7 \%)$ & $37(14.4 \%)$ \\
\hline
\end{tabular}

Aspiration = inhalation of material into the airway below the level of the true vocal cords, with acute symptoms (cough or gag reflex as the bolus passed into the trachea), silent aspiration = aspiration occurring in the absence of acute symptoms 
Table 4 Recommendation for further treatment in 553 patients after initial FEES

\begin{tabular}{lll}
\hline & $\begin{array}{l}\text { Patients after transoral or } \\
\text { transnasal tracheal } \\
\text { intubation }(n=295)\end{array}$ & $\begin{array}{l}\text { Patients with indwelling } \\
\text { tracheotomies }(n=258)\end{array}$ \\
\hline Non-oral feeding (naso-gastric tube) \pm logopedic (functional) therapy & $144(48.8 \%)$ & $\begin{array}{l}131(50.8 \%) \\
46(17.8 \%)\end{array}$ \\
Indication for PEG & $27(9.1 \%)$ & $\mathrm{NA}$ \\
Indication for non-oral feeding plus tracheotomy & $35(11.9 \%)$ & $22(8.5 \%)$ \\
Oral feeding \pm logopedic (functional) therapy & $89(30.2)$ & $\mathrm{NA}$ \\
Decannulation and oral feeding \pm logopedic (functional) therapy & & $59(22.9 \%)$ \\
\hline
\end{tabular}

$P E G$ percutaneous endoscopic gastrostomy, NA not applicable

\section{Discussion}

Aspiration is a common event. The North American Summit on Aspiration in the Critically Ill Patient, in a consensus statement published in 2002 [5], estimates the frequency of aspiration in various patient populations is as follows:

- $45 \%$ in normal individuals during sleep

- $70 \%$ in patients with impaired consciousness

- $0-40 \%$ in patients on ETF

- $50-75 \%$ in patients with endotracheal tubes

What determines the clinical consequences of an aspiration event are host factors (age, immune status, underlying disease process, and comorbidities) and the nature of the aspirate. The morbidity and mortality associated with aspiration (with or without subsequent pneumonia) is difficult to ascertain and may vary greatly depending on the patient population that is under study [5].

Despite these general considerations, aspiration is generally accepted as a leading cause of nosocomial pulmonary infection in the ICU [6].

Ajemian reported a 56\% (27/48) incidence of swallowing dysfunction following prolonged intubation; 12 (25\%) of 48 patients were silent aspirators [7]. In a study by Leder et al. [8], FEES demonstrated swallowing dysfunction in $33 \%$ of critically ill trauma patients after prolonged intubation, with $20 \%$ having silent aspiration. In a recent study by El Solh and co-workers, aspiration was documented in 52\% of ICU patients aged over 65 years and in $36 \%$ of those up to age 65 . No significant difference in the co-morbidity index and the length of mechanical ventilation was found between aspirators and non-aspirators [4].

Non-specific methods to monitor for and detect aspiration are usually subjective, not standardized or validated, and inaccurate, and there are few outcome valid data. Blue dye has been commonly used to color the feeding solutions, but this method is insensitive and non-specific for assessing aspiration and pneumonia. Testing of pharyngeal secretions with glucose oxidase strips also is not standardized and is too insensitive and non-specific to test for aspiration of feeding solution. The best single test for aspiration remains undetermined to date. Therefore, clinical studies are needed to determine the potential role of particular tests suited for specific indications [9].

Video-fluoroscopy has traditionally been accepted as the "gold standard" for evaluation of a swallowing disorder for the comprehensive information it provides. However, it is not very efficient and accessible in certain clinical and practical situations. This is particularly true for critically ill patients treated at ICUs, who are almost invariably bedridden.

FEES has been shown to be safe and effective for assisting in swallowing evaluation, and in therapy as a visual display to help patients learn various swallowing manoeuvres. $[4,10-13]$. It can be used in a wide variety of settings, ranging from office evaluation of swallowing, inpatient and outpatient services to critically ill patient treated at the ICU [14-20].

FEES is not just a screening procedure, it can achieve a complete assessment of the pharyngeal stage of swallowing. It includes five components: assessment of structural changes in the larynx and pharynx, assessment of movement and sensation, assessment of secretions management, direct visualization of swallowing function for food and liquid, and response to therapeutic interventions [12-15, 17].

Aviv and co-workers suggested combining FEES with sensory testing of the laryngeal and pharyngeal mucosa using discrete pulses of air delivered to the epithelium innervated by the internal branch of the superior laryngeal nerve (FEESST: Fiberoptic endoscopic evaluation of swallowing with sensory testing). They performed 1,340 consecutive FEESST procedures on 1,076 adult patients with dysphagia, among them 172 examinations in critically ill patients at the ICU (mostly following cardiac surgery). The authors concluded that FEESST for the purpose of swallowing function can be done safely and yields a $96.5 \%$ success rate in terms of completed examinations $[19,21]$.

Our experience suggests that FEES can be performed easily at the bedside of ICU patients. It is associated with minimal complications for those patients recently liberated from mechanical ventilation, and for patients with indwelling tracheotomy treated at the ICU. However, we cannot 
determine from our data whether adopting FEES prevented aspiration pneumonia. Barquist et al. [22] conducted a randomized, prospective clinical trial of FEES versus routine clinical management in 70 patients after prolonged intubation. The addition of a FEES examination did not change the incidence of aspiration or postextubation pneumonia. Larger prospective randomized trials of FEES versus conventional patient management will be required to determine whether a difference in the rate of post-extubation pneumonia can be demonstrated. However, in our study, the observations recorded during FEES had immediate impact on the further treatment of patients: prolonged non-oral feeding via a naso-gastric tube was initiated in $49.7 \%$ of patients, percutaneous endoscopic gastrostomy in $13.2 \%$, and an additional tracheotomy to prevent aspiration in $6.3 \%$. On the other hand, 81 out of 258 pre-existing tracheotomies were immediately closed, and $30.7 \%$ of all 553 patients with endoscopy-proven aspiration could be treated with the immediate onset of an oral diet and compensatory treatment procedures.

After assessing the critically ill patient for risk of aspiration, the clinician still must decide if the patient is ready to be fed. The goal is to identify critically ill patients who are likely to tolerate enteral nutrition and attempt to minimize complications [23]. FEES is certainly a versatile tool in achieving timely and appropriate clinical decisions in patients at risk for aspiration-related morbidity.

In contrast to most of the recently published material on FEES in critically ill patients, we report our observation in a large proportion of patients with indwelling tracheotomies (258 out of 553, i.e., $46.7 \%$ ) at the time of the examination. Tracheotomy is a commonly performed procedure in ventilator dependent patients. Many critical care practitioners believe that performing a tracheostomy early in the postinjury period decreases the length of ventilator dependence as well as having other benefits such as better patient tolerance and lower respiratory dead space [24]. Table 4 demonstrates that silent aspiration as well as clinically overt aspiration was more frequently encountered in patients with tracheotomies as compared to those that had previously been intubated via an oral or transnasal route. However, the clinical impressions that a tracheotomy or tracheotomy tube increases aspiration risk or that decannulation results in improved swallowing function have not been supported by a recent study. Rather, the need for a tracheotomy indicated comorbidities (e.g. respiratory failure, trauma, stroke, advanced age, reduced functional reserve, and medications used to treat the critically ill) that by themselves predispose patients for dysphagia and aspiration [25]. These data confirmed similar findings previously published by same authors: Neither the presence of a tracheotomy tube nor decannulation affected aspiration status in early, postsurgical head and neck cancer patients. In the acute care setting, no causal relationship between tracheotomy and aspiration status was exhibited [26].

Our results allow no conclusion regarding the impact of tracheotomies on deglutition, since the initial decision to perform a tracheotomy had not been randomly assigned to patients in our two subgroups. The decision to perform tracheotomies was not consistent over different ICUs and largely depended on the anticipated length of assisted ventilation. In our study, FEES was used to determine the need for maintaining tracheotomy, and was accepted by ICU physicians as an important criterion in deciding to close tracheotomies following assisted ventilation.

\section{Conclusion}

After the introduction of a standardized endoscopy protocol for critically ill patients considered being at risk for silent aspiration, ICU physicians soon requested FEES routinely for their patients.

FEES in critically ill patients allows for a rapid evaluation of deglutition, for targeted further diagnostic procedures if needed, and for the immediate initiation of symptom-related rehabilitation or for an early resumption of oral feeding. FEES is now accepted at our institution as an important tool in achieving timely and appropriate clinical decisions for ICU-patients at risk for aspiration-related morbidity.

Laryngologists should be encouraged to offer FEES procedures to responsible coordinators of ICUs.

\section{References}

1. Shifrin RY, Choplin RH (1996) Aspiration in patients in critical care units. Radiol Clin North Am 34:83-96

2. Patel PJ, Leeper KV Jr, McGowan JE Jr (2002) Epidemiology and microbiology of hospital-acquired pneumonia. Semin Respir Crit Care Med 23:415-425

3. Moore FA (2002) Treatment of aspiration in intensive care unit patients. JPEN J Parenter Enteral Nutr 26:S69-S74 (discussion S74)

4. El Solh A, Okada M, Bhat A, Pietrantoni C (2003) Swallowing disorders post orotracheal intubation in the elderly. Intensive Care Med 29:1451-1455

5. McClave SA, DeMeo MT, DeLegge MH, DiSario JA, Heyland DK, Maloney JP, Metheny NA, Moore FA, Scolapio JS, Spain DA, Zaloga GP (2002) North american summit on aspiration in the critically ill patient: consensus statement. JPEN J Parenter Enteral Nutr 26:S80-S85

6. d'Escrivan T, Guery B (2005) Prevention and treatment of aspiration pneumonia in intensive care units. Treat Respir Med 4:317-324

7. Ajemian MS, Nirmul GB, Anderson MT, Zirlen DM, Kwasnik EM (2001) Routine fiberoptic endoscopic evaluation of swallowing following prolonged intubation: implications for management. Arch Surg 136:434-437

8. Leder SB, Cohn SM, Moller BA (1998) Fiberoptic endoscopic documentation of the high incidence of aspiration following extubation in critically ill trauma patients. Dysphagia 13:208-212 
9. DiSario JA (2002) Future considerations in aspiration pneumonia in the critically ill patient: what is not known, areas for future research, and experimental methods. JPEN J Parenter Enteral Nutr 26:S75-S78 (discussion S79)

10. Flaksman H, Ron Y, Ben-David N, Cinamon U, Levy D, Russo E, Sokolov M, Avni Y, Roth Y (2006) Modified endoscopic swallowing test for improved diagnosis and prevention of aspiration. Eur Arch Otorhinolaryngol 263:637-640

11. Langmore SE (1996) Dysphagia in neurologic patients in the intensive care unit. Semin Neurol 16:329-340

12. Langmore SE (2003) Evaluation of oropharyngeal dysphagia: which diagnostic tool is superior? Curr Opin Otolaryngol Head Neck Surg 11:485-489

13. Langmore SE, Schatz K, Olson N (1991) Endoscopic and videofluoroscopic evaluations of swallowing and aspiration. Ann Otol Rhinol Laryngol 100:678-681

14. Langmore SE (1998) Role of flexible laryngoscopy for evaluating aspiration. Ann Otol Rhinol Laryngol 107:446

15. Langmore SE, Schatz K, Olsen N (1988) Fiberoptic endoscopic examination of swallowing safety: a new procedure. Dysphagia 2:216-219

16. Amin MR, Postma GN (2004) Office evaluation of swallowing. Ear Nose Throat J 83:13-16

17. Aviv JE, Kim T, Sacco RL, Kaplan S, Goodhart K, Diamond B, Close LG (1998) FEESST: a new bedside endoscopic test of the motor and sensory components of swallowing. Ann Otol Rhinol Laryngol 107:378-387

18. Aviv JE, Martin JH, Sacco RL, Zagar D, Diamond B, Keen MS, Blitzer A (1996) Supraglottic and pharyngeal sensory abnormalities in stroke patients with dysphagia. Ann Otol Rhinol Laryngol 105:92-97
19. Aviv JE, Murry T, Zschommler A, Cohen M, Gartner C (2005) Flexible endoscopic evaluation of swallowing with sensory testing: patient characteristics and analysis of safety in 1,340 consecutive examinations. Ann Otol Rhinol Laryngol 114:173-176

20. Winklmaier U, Wust K, Plinkert PK, Wallner F (2007) The accuracy of the modified evans blue dye test in detecting aspiration in head and neck cancer patients. Eur Arch Otorhinolaryngol 264:1059-1064

21. Amin MR, Harris D, Cassel SG, Grimes E, Heiman-Patterson T (2006) Sensory testing in the assessment of laryngeal sensation in patients with amyotrophic lateral sclerosis. Ann Otol Rhinol Laryngol 115:528-534

22. Barquist E, Brown M, Cohn S, Lundy D, Jackowski J (2001) Postextubation fiberoptic endoscopic evaluation of swallowing after prolonged endotracheal intubation: a randomized, prospective trial. Crit Care Med 29:1710-1713

23. Spain DA (2002) When is the seriously ill patient ready to be fed? JPEN J Parenter Enteral Nutr 26:S62-S65 (discussion S65-68)

24. Barquist ES, Amortegui J, Hallal A, Giannotti G, Whinney R, Alzamel H, MacLeod J (2006) Tracheostomy in ventilator dependent trauma patients: a prospective, randomized intention-to-treat study. J Trauma 60:91-97

25. Leder SB, Joe JK, Ross DA, Coelho DH, Mendes J (2005) Presence of a tracheotomy tube and aspiration status in early, postsurgical head and neck cancer patients. Head Neck 27:757761

26. Leder SB, Ross DA (2000) Investigation of the causal relationship between tracheotomy and aspiration in the acute care setting. Laryngoscope 110:641-644 